\title{
String Structures on Loop Bundles
}

\author{
R. Coquereaux ${ }^{\star}$ and K. Pilch ${ }^{2 \star \star}$ \\ ${ }^{1}$ Gordon McKay Laboratory, Harvard University, Cambridge, MA 02138, USA \\ 2 Department of Mathematics, Massachusetts Institute of Technology, Cambridge, MA 02139, USA
}

\begin{abstract}
Differential geometry and topology of principal loop bundles (bundles of loop groups over loop spaces) are investigated. String structures, defined as bundle extensions corresponding to the central extension of the structure group, do not always exist. Various methods for deriving the obstruction to the existence of string structures are discussed.
\end{abstract}

\section{Introduction}

Recently, Killingback [1] introduced a notion of string structure and suggested that it should play a similar role in defining the Dirac-Ramond operator on the loop space, $L M$, of a compact riemannian manifold, $M$, as the spin structure does in the case of the usual Dirac operator on $M$.

For a principal fibre bundle, $L P$, over $L M$, with a structure loop group, $L G$, the string structure is defined as a bundle extension of $L P$ to $\hat{L} P$, whose structure group is the central extension of $L G$. In cases which are interesting in applications to string theory the bundles on the loop space are obtained from some bundles over the space itself, for example, from the spin bundle of $M$ or some $G$ bundle which corresponds to the background gauge fields on $M$. It has been also observed in [1] that in such cases the topological conditions, which one derives by requiring that a string structure exists, are similar to those that guarantee the anomaly cancellation in the two-dimensional chiral supersymmetric $\sigma$-model on $M$, coupled to gauge and gravitational backgrounds. This turns out to be quite natural once one realizes the role of the supersymmetric $\sigma$-models in defining the Dirac-type operators [2-6]. For example, it is known that the usual Dirac operator corresponds to the supersymme-

* Supported in part by the U.S. Department of Energy under Grant \#DE-FG02-84ER40158 with Harvard University.

Permanent address: Centre de Physique Theorique, section II, CNRS-Luminy, Case 907, F-13288 Marseille, France.

$\star \star$ Supported in part by the NSF Grant \#87-08447 and by the U.S. Department of Energy under Contract \#DE-AC02-76ER-03069.

On leave from the University of Wroclaw, Wroclaw, Poland 
try charge in the Hilbert space of a one-dimensional chiral supersymmetric $\sigma$-model (supersymmetric particle) [3]. In this case the absence of anomalies, from the point of view of canonical quantization, means that one can construct a Hilbert space for the quantized system as the space of sections of some vector bundle associated with the spin bundle on $M$. In other words the absence of anomalies is equivalent to $M$ being a spin manifold $[7,2]$.

This argument can be generalized to one dimension higher $[1,6]$. Upon quantization the supersymmetry charge of the two-dimensional $\sigma$-model will define the Dirac operator on the loop space. If the chiral fermions in the $\sigma$-model are coupled to some gauge bundle with the structure group, $G$, the resulting DiracRamond operator will naturally be coupled to the bundle over the loop space whose structure group is $L G$. It is reasonable to require that the matter fields on which this operator will act should transform under the unitary representation of the structure group. In the case of the Dirac operator, one enlarges the structure group from $S O(n)$ to $\operatorname{Spin}(n)$ to allow for half-integer spin representations. Here, in the case of the Dirac-Ramond operator, one has to enlarge the structure group $L G$ to a central extension since $L G$ has only projective unitary representations [8].

The obstruction to the existence of a string structure on loop bundles is the vanishing of a certain cohomology class, $\Lambda$, in $H^{3}(L M, \mathbf{Z})$. As already pointed out in [1], this class can be related to the class, $\lambda$, in $H^{4}(M, \mathbf{Z})$ which is responsible for the anomalies in the $\sigma$-model $[9,2,10]$. However, although the vanishing of $\lambda$ implies that $\Lambda$ is trivial, the opposite does not need to hold, and, in fact, examples when it happens are known [11].

When $M$ is an orientable riemannian spin-manifold, one can define spinors on $L M$ by using a projective spin representation of an infinite-dimensional orthogonal group [12]. The existence of such spinors on $L M$ is a stronger requirement than ours and will not be discussed here.

The purpose of this paper is to provide various derivations of the condition for the existence of string structures. Since $\Lambda$ is an element in the integer cohomology of $L M$, in general, it may have a torsion part. However, when there is no torsion, it can be expressed in terms of differential forms on the loop space. This is different from the case of spin structures where the obstruction, the second Stiefel-Whitney class in $H^{2}\left(M, \mathbf{Z}_{2}\right)$, can never be written using the de Rham cohomology of $M$. Thus in the free case (no torsion component in $\Lambda$ ) one should be able to prove the existence of a string structure by studying whether a gauge field on $L M$ with a gauge group $L G$ can be "embedded" into a gauge field with the structure group $\hat{L} G$. The mechanism of how this works is that the resulting gauge field with the gauge group $\hat{L} G$ also has components with values in the $U(1)$ part of the Lie algebra of $\hat{L} G$, and these can be used to construct explicitly a 2 -form $H$ on $L M$ such that $\Lambda=d H$. An obvious virtue of this derivation is that it is explicit and that one needs almost nothing beyond elementary properties of gauge fields. A drawback is that it does not capture the torsion phenomena and is more lengthy than an "abstract" topological proof. Indeed the problem of extending structure groups of fibre bundles is rather standard in mathematical literature and can be related to obstruction thory. In our case once one accepts the classification theorem for principal fibre bundles, the derivation of the condition for the existence of string structures is remarkably straightforward. 
The paper is organized as follows. Section 2 contains a brief development of the calculus of differential forms on loop spaces and a discussion of the relation between the cohomology of the loop space and the cohomology of the space itself. In Sect. 3 we gather information about extensions of groups, in particular of loop groups, and discuss the definition of a string structure for a loop bundle. In Sect. 4 we present an explicit derivation of the obstruction using gauge fields on the loop space. For technical reasons we assume here that $M$ is 2-connected, that is, $\pi_{1}(M)=\pi_{2}(M)=0$. The general case is discussed in Sect. 5 using more powerful methods of obstruction theory. In Sect. 6 we collect a handful of typical examples, mostly of bundles over homogenous spaces, in which the existence of string structure can be verified directly. Finally in Appendix A we recall standard facts about the calculation of homotopy groups of loop spaces and fibre bundles, and in Appendix B we describe an explicit construction of line bundles.

\section{Differential Geometry of Loop Spaces}

2.1. General Definitions. Let $X$ be a finite-dimensional smooth manifold and $L X$ the corresponding loop space. Points in $L X$ are smooth maps $\gamma: S^{1} \rightarrow X$. The tangent space, $T_{\gamma} L X$, to $L X$ at $\gamma$ is the space of sections of the vector bundle $\gamma^{*} T X$ over $S^{1}$. A tangent vector $\xi \in T_{\gamma} L X$, which as usual can be considered as an infinitesimal deformation of the loop $\gamma$, is a loop of vectors $\xi(t), t \in S^{1}$, such that $\xi(t) \in T_{\gamma(t)} X$. A $n$-form $\omega$ at $\gamma$ is defined as a $n$-linear antisymmetric map from $T_{\gamma} L X \times \cdots \times T_{\gamma} L X$ into $\mathbf{R}$. The tangent and cotangent bundles over $L X$ are obtained by taking $\bigcup_{\gamma \in L X} T_{\gamma} L X$ and $\bigcup_{\gamma \in L X} T_{\gamma}^{*} L X$, respectively. Vector fields and differential forms are then sections of the corresponding bundles. Our approach will be rather formal and for that reason we will not discuss in detail the problem of defining the proper smooth structure on $L X$ and on bundles over it. An interested reader is referred to the mathematical literature, for example, $[13,14]$.

2.2. Diff $S^{1}$ Action on $L X$. On $L X$ there is a natural action of Diff $S^{1}$, the group of the diffeomorphisms of the circle, given by

$$
\gamma \rightarrow \phi^{*} \gamma, \quad \phi \in \operatorname{Diff} S^{1}, \quad \gamma \in L X .
$$

The Lie algebra of Diff $S^{1}$ can be identified with the space of smooth vector fields on $S^{1}$. For a given smooth function $f(t)$ on the circle, we will denote the corresponding generator of DiffS ${ }^{1}$ by $L(f)=f d / d t$ and the fundamental vector field on $L X$ at $\gamma$, which corresponds to the above action, by $\tilde{L}(f)_{\gamma}$. In terms of the canonical tangent vector $\dot{\gamma} \in T_{\gamma} L X$ given by a loop of vectors tangent to the loop $\gamma, \dot{\gamma}(t)=d \gamma(t) / d t$, we can express the latter as $\tilde{L}(f)_{\gamma}(t)=f(t) \dot{\gamma}(t)$. Note that the vector field $\dot{\gamma}$ itself corresponds to the generator $L(1)$ of the subgroup of constant rotations along the circle.

2.3. Loop Forms on $L X$. Let $\omega$ be a differential form of degree $n$ on $X$. It is convenient to introduce a corresponding $n$-form $\bar{\omega}$ on $L X$, with values in $L \mathbf{R}$, which 
at $\gamma \in L X$ is defined by

$$
\bar{\omega}\left(\gamma ; \xi_{1}, \ldots, \xi_{n}\right)(t)=\omega\left(\gamma(t) ; \xi_{1}(t), \ldots, \xi_{n}(t)\right), \quad \xi_{1}, \ldots, \xi_{n} \in T_{\gamma} L X .
$$

This generalizes to forms $\omega$ which take values in some vector space $V$ and gives forms $\bar{\omega}$ with values in $L V$. We will refer to $\bar{\omega}$ as a loop form corresponding to $\omega$.

For a loop form $\bar{\omega}$ we define its derivative $d \bar{\omega} / d t$ as

$$
\left(\frac{d}{d t} \bar{\omega}\right)\left(\xi_{1}, \ldots, \xi_{n}\right)(t)=\frac{d}{d t} \bar{\omega}\left(\xi_{1}, \ldots, \xi_{n}\right)(t) .
$$

Similarly the integral $\int \bar{\omega}$, which maps $\bar{\omega}$ into an R-valued (or more generally $V$-valued) form on $L X$ is defined by

$$
\left(\int \bar{\omega}\right)\left(\xi_{1}, \ldots, \xi_{n}\right)=\int_{S^{1}} d t \bar{\omega}\left(\xi_{1}, \ldots, \xi_{n}\right)(t) .
$$

Both the derivative and the integral are defined for any forms on $L X$ provided they take values in some loop space of a vector space. An obvious property is $\int d / d t \equiv 0$. Since $d / d t$ and $\int$ are linear operators in the space of values of loop forms, they commute with the usual operations of the differential calculus of forms such as the exterior derivative, $d$, and the contraction with a vector field $\xi, i_{\xi}$, which are defined on $L X$. Consequently they also commute with the Lie derivative along $\xi$, which, as usual, is defined as

$$
\mathscr{L}_{\xi}=d i_{\xi}+i_{\xi} d \text {. }
$$

From the definition (2) and the action of Diff $S^{1}$ in (1) we deduce that loop forms are Diff $S^{1}$ equivariant in the following sense:

$$
\mathscr{L}_{\tilde{L}(f)} \bar{\omega}=L(f) \bar{\omega} .
$$

Here the operator on the left-hand side is the Lie derivative along the fundamental vector field $\tilde{L}(f)$ on $L X$, and $L(f)$ on the right-hand side is the differential operator $f(t) d / d t$ in $L \mathbf{R}$.

2.4. Local Forms and the Evaluation Map. Another important operation is the averaged evaluation map, $E$, which sends $n$-forms on $X$ into $(n-1)$-forms on $L X$. It is defined by

$$
E \omega=\int i_{y} \bar{\omega}
$$

or more explicitly

$$
E \omega\left(\gamma ; \xi_{1}, \ldots, \xi_{n-1}\right)=\int_{S^{1}} d t \omega\left(\gamma(t) ; \dot{\gamma}(t), \xi_{1}(t), \ldots, \xi_{n-1}(t)\right)
$$

Notice that the form $E \omega$ is obtained as follows. First we use the evaluation map $e: S^{1} \times L X \rightarrow X, e(t, \gamma)=\gamma(t)$ to pull back $\omega$ from $X$ to $e^{*} \omega$ on $S^{1} \times L X$ and then integrate it over the fibre $S^{1}$. The forms on $L X$ which are in the image of $E$ will be called local.

All local forms are Diff $S^{1}$ invariant. This follows from the manifest reparametrization invariance of the integral in (3) that defines $E$. Infinitesimally we have

$$
\mathscr{L}_{L(f)} E \equiv 0 .
$$


An obvious remark is that, since not all forms on $L X$ are Diff $S^{1}$ invariant, there exist forms which are not local.

An important property of $E$ is

$$
E d=-d E
$$

Proof.

$$
d E \omega=d \int i_{\dot{\gamma}} \bar{\omega}=\int d i_{\dot{\gamma}} \bar{\omega}=\int\left[\mathscr{L}_{\dot{\gamma}}-i_{\dot{\gamma}} d\right] \bar{\omega}=\int \frac{d}{d t} \bar{\omega}-\int i_{\dot{\gamma}} \overline{d \omega}=0-E d \omega .
$$

This shows that closed forms on $X$ are mapped by $E$ into closed forms on $L X$. Therefore, the complex of closed local forms on $L X$ is a subcomplex of closed forms on $L X$, and $E$ induces a map from the de Rham cohomology of $X$ into the de Rham cohomology of $L X$.

Consider the following example. If $\omega$ is a 1 -form on $X$, then $E \omega$ is a function on $L X$ whose value on loop $\gamma$ is given by the line integral of $\omega$

$$
E \omega(\gamma)=\int_{\gamma} \omega .
$$

This generalizes to higher-degree forms as follows. Let $c_{n}$ be a $n$-chain in $L X$. Then $e\left(S^{1} \times c_{n}\right)$ is a $(n+1)$-dimensional submanifold in $X$ (possibly with singular points). For $a(n+1)$-form $\omega$ in $X$ we have

$$
\int_{c_{n}} E \omega=\int_{e\left(S^{1} \times c_{n}\right)} \omega .
$$

The pairing between forms and chains defined in (6) can be extended to singular chains and cochains (one must simply replace the integrals by abstract pairings), and defines an extension of the averaged evaluation map $E$ to singular cohomology.

2.5. Relation Between the Cohomology of $X$ and $L X$. To understand the relation between the existence of a string structure and the absence of anomalies in the corresponding $\sigma$-model we must know the precise relation between the cohomology of $X$ and $L X$ and, in particular, whether $E$, as a map from $H^{n}(X, *)$ into $H^{n-1}(L X, *)$, is injective. We do not know the complete answer to this question. If $X$ is a simple compact Lie group $G$, then it can be shown that $E$ is an isomorphism between cohomologies of $G$ and $L G$ [8].

From the duality between homology and cohomology, the question whether $E$ is injective is the same as whether $H_{n}(X, *)$ is generated by cycles $c_{n}$ which are of the form $e\left(S^{1} \times c_{n-1}\right)$. This happens, for example, if all $c_{n}$ 's are homologous to homotopy spheres. In such a case we can use an explicit cover of $S^{n}$ with loops which intersect each other only at the north pole (the set of such loops is topologically $S^{n-1}$ ) to construct the corresponding cycle in $L X$. We can apply this observation to the case when $X$ is simply connected. Let us recall that for simply connected $X$ the Hurewicz theorem [15] guarantees that the first nontrivial homology group $H_{k}(X, \mathbf{Z})$ is isomorphic to $\pi_{k}(X)$, and that the next one, $H_{k+1}(X, \mathbf{Z})$, is generated by the image of $\pi_{k+1}(X)$. Therefore, we can conclude that whenever 
$\pi_{1}(X)=0, E$ is an isomorphism in the first dimension where homology is nontrivial and becomes an injection in one dimension higher.

\section{Loop Groups, Loop Bundles and String Structures}

3.1. Loop Groups. The loop group, $L G$, of a finite-dimensional Lie group, $G$, is defined [8] as a group of smooth maps from $S^{1}$ into $G$ with the pointwise multiplication, i.e., $\left(g_{1} g_{2}\right)(t)=g_{1}(t) g_{2}(t), g_{1}, g_{2} \in L G, t \in S^{1}$. The Lie algebra of $L G$ can be identified with the space of loops in Lie $G$ with the commutator

$$
[\xi, \eta](t)=[\xi(t), \eta(t)], \quad \xi, \eta \in \operatorname{Lie} L G, \quad t \in S^{1} .
$$

$L G$ is an infinite-dimensional group and can be given a structure of a smooth Hilbert manifold. An important property of loop groups is that the exponent map Exp: Lie $L G \rightarrow L G$ is locally a homeomorphism. This is not always true for any infinite dimensional group and the notable example when Exp is not a homeomorphism is Diff $S^{1}$ [14]. From the practical point of view a nice exponential map means that we can check a lot of properties at the level of the Lie algebra and then generalize them to the level of the group. In fact, to a large extent differential geometry involving loop groups can be done in a similar way as with finitedimensional groups [16]. We will make use of this in Sect. 4.

3.2. Group Extensions. We say that a group $\hat{H}$ is an extension of a group $H$ if there exists a homomorphism $\varphi$ from $\hat{H}$ onto $H$. Thus to define an extension we must specify the pair: group $\hat{H}$ and epimorphism $\varphi$.

Any extension defines a principal fibration

$$
\operatorname{ker} \varphi=N \rightarrow \quad \begin{aligned}
& \hat{H} \\
& H \simeq \hat{H} / N
\end{aligned}
$$

in which $\hat{H}$ is the total space, $H$ is the base and $N=\operatorname{ker} \varphi \subset \hat{H}$ is the structure group.

We will consider two classes of extensions, group coverings and abelian central extensions.

3.2.1. Group Coverings. This is the case when $N$ is a discrete group. The exact homotopy sequence for the fibration (A.1) ${ }^{1}$ gives

$$
\begin{aligned}
& \pi_{k}(H)=\pi_{k}(\hat{H}), \quad k \geqq 2, \\
& \pi_{1}(H) / \pi_{1}(\hat{H})=N .
\end{aligned}
$$

An important example of a group covering is the universal covering of the group $H$ which is defined by the condition that $\hat{H}$ is simply connected. In this case $\pi_{1}(H)=N$.

\subsubsection{Abelian Central Extensions. In this case $N$ is an abelian group and the group}

\footnotetext{
${ }^{1}$ See Appendix A
} 
multiplication in $\hat{H}$, which, at least locally, is a product space $H \times N$, is of the form

$$
(h, n) \cdot\left(h^{\prime}, n^{\prime}\right)=\left(h h^{\prime}, c\left(h, h^{\prime}\right) n n^{\prime}\right), \quad h, h^{\prime} \in H, \quad n, n^{\prime} \in N,
$$

where $c($,$) is a cocycle. In general c$ must be considered as a section of some bundle; however, if $\hat{H}$ is globally a product $H \times N$, then $c$ is a function, $c: H \times H \rightarrow N$. In order that (7) defines a group multiplication, $c$ must satisfy cocycle conditions, which in the product case can be written explicitly as

$$
\begin{array}{ll}
\text { (i) } & c(1, h)=c(h, 1)=1, \\
\text { (ii) } & c\left(h, h^{\prime} h^{\prime \prime}\right) c\left(h^{\prime}, h^{\prime \prime}\right)=c\left(h, h^{\prime}\right) c\left(h h^{\prime}, h^{\prime \prime}\right) .
\end{array}
$$

Note that locally $\varphi(h, n)=h$ and $N$ is a subgroup in the center of $\hat{H}$.

3.3. Central Extensions of Loop Groups. In the following we assume that $G$ is simple and simply connected.

3.3.1. General Definitions. We wish to describe briefly an important class of central extensions of $L G$ by $U(1)$. For a more detailed discussion and proofs, the standard reference is [8].

On the level of Lie algebras a $U(1)$ extension $\hat{L} G$ of $L G$ can be written as Lie $\hat{L} G=\operatorname{Lie} L G \oplus \mathbf{R}$ with the Lie bracket

where

$$
[(\xi, \alpha),(\eta, \beta)]=([\xi, \eta], \omega(\xi, \eta)) \quad \xi, \eta \in \operatorname{Lie} L G, \quad \alpha, \beta \in \mathbf{R},
$$

$$
\omega(\xi, \eta)=\frac{1}{2 \pi} \int_{0}^{2 \pi} d t\left\langle\xi(t), \frac{d}{d t} \eta(t)\right\rangle
$$

is a cocycle on Lie $L G$. The cocycle condition for the group multiplication as given in (7) implies that

$$
\omega([\xi, \eta], \zeta)+\omega([\eta, \zeta], \xi)+\omega([\zeta, \xi], \eta)=0, \quad \xi, \eta, \zeta \in \text { Lie } L G .
$$

However, as we will see later not all cocycles on Lie $L G$ can be obtained from some cocycle in $L G$.

All the cocycles, and thus all central extensions of Lie $L G$, are completely determined by the choice of a symmetric invariant form $\langle$,$\rangle in Lie G$.

The Lie algebra cocycle $\omega$ can also be considered as a left-invariant 2-form on $L G$,

$$
\omega\left(g ; d L_{g} \xi, d L_{g} \eta\right)=\omega(\xi, \eta), \quad g \in L G, \quad \xi, \eta \in \operatorname{Lie} L G .
$$

From the cocycle condition (9) it follows that this form is closed and thus defines a cohomology class in $H^{2}(L G, \mathbf{R})$.

The fundamental theorem is that the extension of Lie $L G$ defined by $\omega$ can be integrated to an extension of Lie groups if and only if the cohomology class of $\omega / 2 \pi$ is integral, i.e., $\omega$ integrated over any closed 2-cycle gives a multiple of $2 \pi$.

3.3.2. Explicit Construction of the U(1) Central Extension of LG. The Lie group (if it exists) corresponding to Lie $\hat{L} G$ is a $U(1)$ bundle over $L G$ and $\omega / 2 \pi$ represents the first Chern class of this bundle. Since $L G$ is a simply connected space (A.2), $\omega$ 
determines this bundle completely. The situation here is analogous to finite dimensions. Using the construction described in Appendix B we can give the following explicit representation for $\hat{L} G$ [8]. One considers the set of triples $[g, l(g), u]$, where $g \in L G, l(g)$ is a path in $L G$ from the unit element of $L G$ to $g$, and $u \in U(1)$. In this set one can define an equivalence relation which identifies $[g, l(g), u]$ with $\left[g^{\prime}, l^{\prime}\left(g^{\prime}\right), u^{\prime}\right]$ if and only if $g=g^{\prime}$ (i.e., $g(t)=g^{\prime}(t)$ for all $t$ ) and $u=\Delta\left(l^{\prime} * l^{-1}\right) u^{\prime}$. Here $l^{\prime} * l^{-1}$ denotes a closed path in $L G$ obtained by going first along $l^{\prime}$ and then back along $l$, and

$$
\Delta\left(l^{\prime} * l^{-1}\right)=e^{i j \omega}
$$

where $\sigma$ is an arbitrary surface in $L G$ bounded by $l^{\prime} * l^{-1}$. As a set $\hat{L} G$ is then the set of all triples divided by the relation we have just discussed. The group multiplication in $\hat{L} G$ is

$$
\left[g_{1}, l_{1}, u_{1}\right] \cdot\left[g_{2}, l_{2}, u_{2}\right]=\left[g_{1} g_{2}, l_{1} * g_{1} l_{2}, u_{1} u_{2}\right] .
$$

It is straightforward to verify that this is well defined with respect to the equivalence relation. The projection on the first entry in $[,$,$] is the homomorphism \varphi$ onto $L G$ whose kernel is the center $U(1)$.

3.3.3. Basic Central Extension when $G$ is Simple. When $G$ is simple all the $U(1)$ central extensions of $L G$ can easily be classified. Since $G$ is simple, all bilinear invariant forms on Lie $G$ are proportional (all bi-invariant metrics on $G$ are proportional), and there exists a smallest one for which $\omega / 2 \pi$ is integral. This form is called basic and is explicitly given by

$$
\langle\xi, \eta\rangle=\frac{1}{2 n_{G}}\langle\xi, \eta\rangle_{G},
$$

where $\langle,\rangle_{G}$ is the Killing form and $n_{G}$ is the dual Coxeter number [17]. The corresponding central extension $\hat{L} G$ has two important properties [8]:

(i) It is universal in the sense that any central extension $\mathscr{E}$ of $L G$ by an abelian group $N$ can be obtained from it by specifying a homomorphism $\rho: U(1) \rightarrow N$. Then $\mathscr{E}=\widehat{L} G \times_{U(1)} N$, where we identify pairs of the form $(\hat{g}, k) \simeq\left(\hat{g} \alpha, \rho\left(\alpha^{-1}\right) k\right)$, $\hat{g} \in \hat{L} G, \alpha \in U(1) \subset \hat{L} G$ and $k \in N$.

(ii) $\hat{L} G$ is 2 -connected, i.e., $\pi_{1}(\hat{L} G)=\pi_{2}(\hat{L} G)=0$.

The second property easily follows from the homotopy sequence for the fibration $\hat{L} G(L G, U(1))$, (A.4). It is convenient to think about the basic central extension $\hat{L} G$ as the $U(1)$ bundle on $L G$ with the "lowest monopole charge."

3.3.4. Central Extensions with $G$ Semi-Simple. When $G$ is not simple, but instead has several simple factors, $G=G_{1} \times \cdots \times G_{n}$, there also exists a basic central extension which is universal, but it is an extension by a $n$-dimensional torus rather than a single $U(1)$ [8]. In the context of string theory the extensions of non-simple groups that one considers are extensions by a single $U(1)$ with a cocycle of the form

$$
\omega\left(\left(\xi_{1}, \ldots, \xi_{n}\right),\left(\eta_{1}, \ldots, \eta_{n}\right)\right)=\sum_{i=1}^{n} m_{i} \omega^{(i)}\left(\xi_{i}, \eta_{i}\right), \quad \xi_{i}, \eta_{i} \in \operatorname{Lie} L G_{i},
$$


where $\omega^{(i)}$ are the basic cocycles in Lie $L G_{i}$, and $m_{i}$ are integers. These extensions can be obtained from the basic one by choosing $\rho: U(1)^{n} \rightarrow U(1)$ of the form $\rho\left(\alpha_{1}, \ldots, \alpha_{n}\right)=\alpha_{1}^{m_{1}} \ldots \alpha_{n}^{m_{n}}$.

3.3.5. Relation Between $H^{2}(L G)$ and $H^{3}(G)$. As we already mentioned in Sect. 2.5, the averaged evaluation map $E$ is an isomorphism between cohomology groups of $G$ and $L G$. The generator of $H^{3}(G)$ is represented by an invariant 3-form $\sigma$ given by

$$
\sigma(\xi, \eta, \zeta)=\frac{1}{8 \pi^{2}}\langle\xi,[\eta, \zeta]\rangle, \quad \xi, \eta, \zeta \in \operatorname{Lie} G
$$

The image of $\sigma$ in $L G$ under $E$ is a 2 -form $E \sigma$ on $L G$ given by

$$
(E \sigma)\left(g ; d L_{g} \xi, d L_{g} \eta\right)=\int_{0}^{2 \pi} d t \sigma\left(d L_{g-1} \dot{g}(t), \xi(t), \eta(t)\right) .
$$

However, $E \sigma$ is neither left nor right invariant. It is nevertheless cohomologous (as it should be) to the left-invariant basic form $\omega$ in $L G$ defined in (10). The precise relation between $\omega$ and $E \sigma$ is [18]

$$
\frac{1}{2 \pi} \omega=E \sigma+d \beta
$$

where the 1 -form $\beta$ is given by

$$
\beta\left(g ; d L_{g} \xi\right)=-\frac{1}{8 \pi^{2}} \int_{0}^{2 \pi} d t\left\langle d L_{g^{-1}} \dot{g}(t), \xi(t)\right\rangle .
$$

3.3.6. Central Extensions when $G$ is not Semi-Simple. When $G$ is not semi-simple, all the central extensions by $U(1)$ can also be classified. However, it is much more complicated than in the simply connected case [8]. Firstly, the loop group $L G$ is not connected, and extensions of the connected component do not necessarily extend to the extension of the whole group. Secondly, the connected component of the identity, $L G_{0}$, is not simply connected and, as a result, $U(1)$ bundles over it are not completely determined by the curvature 2 -form, i.e., by the cocycle in the Lie algebra.

3.4. Loop Bundles. Let $P(M, G, \pi)$ be a principal fibre bundle with total space $P$, base $M$, structure group $G$, and projection $\pi: P \rightarrow M$. The corresponding loop bundle, $L P\left(L M, L G, \pi_{L}\right)$, is obtained by considering the space of loops in $P$. If $p$ is a loop in $P$, then $\pi_{L}(p)=\pi \circ p$ is a loop in $M$. It is obvious that any two loops in $P$ that project onto the same loop in $M$ are related by an element in $L G$. The action of $L G$ on $L P$ is free and we conclude that $L P$ is a principal $L G$-bundle over $L M$.

The action of DiffS $S^{1}$ on $L P$ as a loop space (see Sect. 2.2) defines a lift of the action on the base, $L M$, and is an action of a group of twisted automorphism of the bundle $L P$ in the sense that

$$
\phi(p g)=\phi(p) \phi(g) \quad p \in L P, \quad g \in L G, \quad \phi \in \operatorname{Diff}^{1} .
$$

Note that usually an automorphism $\psi$ would act by $\psi(p g)=\psi(p) g$. 
3.5. String Structures in Loop Bundles. Let $L P(L M, L G)$ be a principal loop bundle and $\hat{L} G$ a $U(1)$-central extension of $L G$ with the projection $\varphi: \hat{L} G \rightarrow L G$. We will say that $L P(L M, L G)$ admits a string structure [1] if there exists a principal bundle $\hat{L} P(L M, \hat{L} G)$, with base $L M$ and the structure group $\hat{L} G$, together with a map $\Phi: \hat{L} P \rightarrow L P$ such that $\Phi(\hat{p} \hat{g})=\Phi(\hat{p}) \varphi(\hat{g})$, for all $\hat{p} \in \hat{L} P$ and $\hat{g} \in \hat{L} G$. More precisely we require the following diagram to be commutative:

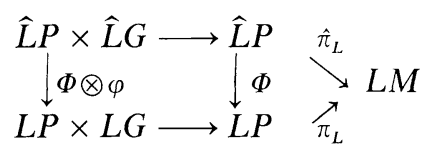

Remarks.

1. The notion of string structure is relative to the bundle $L P$ and the extension $\hat{L} G$. It can be defined for any $L G$ bundle, not necessarily a loop bundle. Most of our discussions and, in particular the existence conditions that will be described in Sects. 4 and 5, are valid in the general case. If $G$ is simple, we will usually take $\widehat{L} G$ to be the basic central extension. For non-simple groups we may also consider string structures corresponding to more general toroidal extensions of $L G$.

2. We will say that a bundle $P(M, G)$ has a string structure if its loop bundle has one, and that a spin manifold $M$ admits a string structure if its spin bundle does. The latter is in analogy with the similar convention for spin structures.

3. The right action of $U(1) \subset \widehat{L} G$ on $\hat{L} P$ gives it a structure of a $U(1)$ bundle over $L P$. This bundle, when considered over the fibres of $L P(L M, L G)$, is isomorphic with the bundle $\hat{L} G(L G, U(1))$. We will make frequent use of this observation.

\section{Gauge fields on Loop Spaces and the Existence of String Structure}

In this section we assume that $\pi_{i}(M)=0, i \leqq 2$ and $\pi_{i}(G)=0, i \leqq 1$.

4.1. Gauge Fields as Principal Connections. Let $P(M, G)$ be a principal fibre bundle with a principal connection $A$. We recall that $A$ is a Lie $G$ valued 1 -form on the total space $P$ which satisfies [19]

$$
\begin{aligned}
\text { (i) } & A\left(\widetilde{\xi}_{p}\right)=\xi, \quad \xi \in \operatorname{Lie} G, \\
\text { (ii) } R_{g}{ }^{*} A & =\operatorname{Ad} g^{-1} A, \quad g \in G,
\end{aligned}
$$

where $\tilde{\xi}_{p}$ is the fundamental vector at $p \in P$ corresponding to $\xi$, and $R_{g}$ is the right action of $G$ on $P$.

The curvature 2-form, $F$, is obtained by

$$
F=d A+\frac{1}{2}[A, A]
$$

and satisfies

$$
\begin{aligned}
& \text { (i) } i_{\tilde{\xi}_{p}} F=0, \\
& \text { (ii) } R_{g} * F=\operatorname{Ad} g^{-1} F .
\end{aligned}
$$

Finally, let us recall that in order to obtain the description of the gauge field more familiar to physicists, one must choose a set of local trivializations of the 
bundle $P$, which consists of covering $\left\{\mathscr{U}_{\alpha}\right\}$ of $M$ with open sets together with local sections $\sigma_{\alpha}: \mathscr{U}_{\alpha} \rightarrow P$ such that $\pi \circ \sigma_{\alpha}=$ id. The gauge potential $A^{(\alpha)}$ on the open set $\mathscr{U}_{\alpha}$ is then obtained by pulling back $A$ from $P$ onto $\mathscr{U}_{\alpha}$, namely $A^{(\alpha)}=\sigma_{\alpha}^{*} A$. Local sections are related by $\sigma_{\alpha}(x)=\sigma_{\beta}(x) g_{\alpha \beta}(x), x \in \mathscr{U}_{\alpha} \cap \mathscr{U}_{\beta}$, where $g_{\alpha \beta}: \mathscr{U}_{\beta} \cap \mathscr{U}_{\beta} \rightarrow G$ are the transition functions. Properties in (16) and (17) then imply the familiar transformation laws for gauge potentials and field strengths.

4.2. Chern-Simons Forms on $P$ and LP. The fibres of $P$ can be identified (up to the choice of the origin) with the group $G$. Thus on each fibre there is a third cohomology class corresponding to the cohomology class of $G$ discussed in Sect. 3.3.5. The form which represents this cohomology class on the fibre can be specified by its values on fundamental vector fields and will be called $\sigma$. We can choose, for example,

$$
\sigma\left(\tilde{\xi}_{1}, \tilde{\xi}_{2}, \tilde{\xi}_{3}\right)=\sigma\left(\xi_{1}, \xi_{2}, \xi_{3}\right), \quad \xi_{1}, \xi_{2}, \xi_{3} \in \operatorname{Lie} G,
$$

where the right-hand-side is given by (12).

It is standard that, using a connection $A$, one can extend $\sigma$ as a Chern-Simons form [20] $\omega_{3}$ to the total space of the bundle

$$
\omega_{3}=\frac{1}{8 \pi^{2}}\left\langle A, F-\frac{1}{6}[A, A]\right\rangle .
$$

Here the product $\langle$,$\rangle of Lie algebra-valued forms should be understood as follows.$ Let $T_{i}$ be the generators of Lie $G$. Then $\langle A, F\rangle=A^{i} \wedge F^{j}\left\langle T_{i}, T_{j}\right\rangle$, and similarly for the second term.

Using (16) and (17) for $A$ and $F$, it is easy to check that $\omega_{3}$ restricted to the fibre indeed reproduces $\sigma$. Moreover, $d \omega_{3}$ is a basic form, that is, there exists a 4-form $\lambda$ on $M$ such that

$$
d \omega_{3}=\pi^{*} \lambda .
$$

To show this, one must check that $i_{\xi} d \omega_{3}=0$, which follows from (i) of (16), and that $\mathscr{L}_{\xi} d \omega_{3}=0$, which follows from (16), (17) and ad-invariance of the form $\sigma$ in Lie $G$. Note that $\lambda$ is necessarily a closed form and thus represents a cohomology class of the base, which is called a transgression of the cohomology class of the fibre represented by $\sigma$. In our case we can choose $\lambda=-\left(1 / 8 \pi^{2}\right)\langle F, F\rangle$. When $G$ is $\operatorname{Spin}(n), \lambda=-\frac{1}{2} p_{1}$, where $p_{1}$ is the Pontrjagin class.

Let $L P(L M, L G)$ be the loop bundle obtained from $P(M, G)$ as described in Sect. 3.4. Consider the 2-form $E \omega_{3}$ on $L P$. Using (5) and (20) we find

$$
d E \omega_{3}=-E d \omega_{3}=-E \pi^{*} \lambda=-\pi_{L}^{*} E \lambda .
$$

The 2-form $E \omega_{3}$ is equal to $E \sigma$ when evaluated on fundamental vectors at $p \in L P$. Thus upon restriction to the fibres of $L P, E \omega_{3}$ represents the cohomology class of the form $\omega$ on $L G$ that was used in Sect. 3.3.1 to define the central extension $\hat{L} G$. In the case of $L G$ we had $(1 / 2 \pi) \omega=E \sigma+d \beta$. Here we will show that one can introduce a 2 -form $\Psi_{2}$ whose restriction to the fibre is exactly equal to the form $\omega$, and $(1 / 2 \pi) \Psi_{2}=E \omega_{3}+d r$, where $r$ is some 1-form on $L P$. To achieve this we use the natural connection in $L P$ given by the loop form $\bar{A}$, where $A$ is some connection form 
in $P$. Using (16) and (17) it is easy to check that the following form defined on $L P$.

$$
\Psi_{2}=\frac{1}{2 \pi} \int_{0}^{2 \pi} d t\left(\frac{1}{2}\left\langle\bar{A}, \frac{d}{d t} \bar{A}\right\rangle-\langle\bar{F}, \bar{A}(\dot{\gamma})\rangle\right)
$$

satisfies $\Psi_{2}\left(\tilde{\xi}_{p}, \tilde{\eta}_{p}\right)=\omega(\xi, \eta)$.

We saw in Sect. 3.3.5 that on $L G$ the form $\omega$ was cohomologous to $E \sigma$. Let us show that the same holds for their extensions to $L P$. Consider a 1-form, $r$, on $L P$,

$$
\Upsilon=-\frac{1}{8 \pi^{2}} \int_{0}^{2 \pi} d t\langle\bar{A}, \bar{A}(\dot{\gamma})\rangle .
$$

Let us show that

$$
\frac{1}{2 \pi} \Psi_{2}=E \omega_{3}+d r .
$$

The proof is an elementary use of formulae from Sect. 2. We shall write it out in some detail to point out the importance of $\bar{A}$ being a loop form on $L P$. We have

$$
\begin{aligned}
-8 \pi^{2} d \Upsilon & =d \int\left\langle\bar{A}, i_{\dot{\gamma}} \bar{A}\right\rangle=\int\left[\left\langle d \bar{A}, i_{\dot{\gamma}} \bar{A}\right\rangle-\left\langle\bar{A},\left(\mathscr{L}_{\dot{\gamma}}-i_{\dot{\gamma}} d\right) \bar{A}\right\rangle\right] \\
& =\int\left[-\left\langle\bar{A}, \frac{d}{d t} \bar{A}\right\rangle+2\left\langle d \bar{A}, i_{\dot{\gamma}} \bar{A}\right\rangle-i_{\dot{\gamma}}\langle d \bar{A}, \bar{A}\rangle\right] \\
& =-4 \pi \Psi_{2}+8 \pi^{2} E \omega_{3} .
\end{aligned}
$$

From (20), (21) and (24) we obtain

$$
\frac{1}{2 \pi} d \Psi_{2}=d\left(E \omega_{3}+d r\right)=-\pi_{L}^{*} E \lambda .
$$

Remark. On $L P, E \omega_{3}$ is explicitly given by

$$
E \omega_{3}=-\frac{1}{8 \pi^{2}} \int i_{\hat{\gamma}}\left(\left\langle\bar{A}, \bar{F}-\frac{1}{6}[\bar{A}, \bar{A}]\right\rangle\right), \quad \gamma \in L P .
$$

Notice that the right hand side of this expression is well defined if we replace $\bar{A}$ by any connection $\mathscr{A}$ in $L P$. This defines a form on $L P$ which we will call $\omega_{2}$. In the same way forms $\Psi_{2}$ and $Y$ can be defined for any connection $\mathscr{A}$ in $L P$. Moreover, we can define $\omega_{2}, \Psi_{2}$ and $Y$ for any $L G$ bundle over $L M$, without assuming that it comes from some $G$ bundle over $M$, provided it admits a lift of the $S^{1}$ action on the base to the twisted group of automorphisms (3.4). In this case we simply replace $\dot{\gamma}$ in (22), (23) and (26) by the fundamental vector field of the $S^{1}$-action. Then one can check that $d \Psi_{2}$ is a basic form, that is

$$
\frac{1}{2 \pi} d \Psi_{2}=-\pi_{L}^{*} \Lambda,
$$

where $\Lambda$ (not to be confused with $\lambda$ ) is a closed form on $L M$. However, in this more general situation one cannot prove that, for any connection $\mathscr{A}, \Psi_{2}$ is cohomologous to $\omega_{2}$. This can be seen from our explicit calculation after (24) in which we used the fact that the connection $\bar{A}$ was DiffS ${ }^{1}$-equivariant in the sense of Sect. 2.3. In order 
that the relation $(1 / 2 \pi) \Psi_{2}=\omega_{2}+d \beta$ holds for an arbitrary connection $\mathscr{A}$ we must restrict the class of allowed connections to those which are given by $S^{1}$ equivariant forms, that is, they satisfy

$$
\mathscr{L}_{\dot{\gamma}} \mathscr{A}=\frac{d}{d t} \mathscr{A} .
$$

For such forms one can also show that the cohomology class of $\Lambda$ does not depend on the choice of the equivariant connection used to define it. We can thus consider $(1 / 2 \pi) \Psi_{2}$ as a generalization of the "Chern-Simons" forms to loop bundles, and $\Lambda$ is then the generalization of the Pontrjagin class. Of course, when the $L G$ bundle comes from some $G$ bundle, $\Lambda$ is cohomologous to $E \lambda$.

4.3. Existence of String Structure. We will now discuss in the framework of gauge fields on $L M$ the existence of string structure for the bundle $P(M, G)$. First we will prove that the vanishing of $\Lambda$ is a necessary condition for the existence of a string structure. The strategy is as follows. Assuming that an extension $\hat{L} P$ exists, we extend the gauge field in $L P$ to some gauge field in $\widehat{L} P$. Then we use the $U(1)$ component of this gauge field to construct explicitly a 2-form $H$ in $L M$ such that $d H=\Lambda$. We will first discuss the case where $G$ is simple and $\hat{L} G$ is the basic central extension.

Let $\hat{L} P(L M, \hat{L} G)$ be an extension of $L P(L M, L G)$ which defines a string structure of the bundle $P(M, G)$. As we discussed before, $\hat{L} P$ can also be viewed as a $U(1)$ bundle, $\hat{L} P(L P, U(1))$, over $L P$ that is isomorphic with the central extension $\hat{L} G(L G, U(1))$ over the fibres of $L P$.

Corresponding to the decomposition of the Lie algebra of $\hat{L} G$, Lie $\hat{L} G=$ Lie $L G \oplus \mathbf{R}$, there is a decomposition of any principal connection $\hat{\mathscr{A}}$ in $\hat{L} P$ into its $L G$ and $U(1)$ parts. Let $\hat{\mathscr{A}}=(\mathscr{A}, \theta)$. A natural question that arises is whether it is possible to combine arbitrary connections $\mathscr{A}$ in $L P(L M, L G)$ and $\theta$ in $\hat{L} P(L P, U(1))$ in such a way that they form a connection $\hat{A}$ in $\hat{L} P(L M, \hat{L} G)$. In general the answer is negative, since in order to be part of a connection in $\widehat{L} P(L P, \widehat{L} G), \theta$ cannot be arbitrary along the fibres of $L P$. In fact, from the infinitesimal form of (ii) in (16) and the commutation relations of $\operatorname{Lie} \hat{L} G$, we find that

$$
\mathscr{L}_{\hat{\tilde{\xi}}} \theta=-[\xi, \mathscr{A}]_{U(1)}=-\omega(\xi, \mathscr{A}),
$$

where $\hat{\xi}$ is the fundamental vector field in $\hat{L} P$ corresponding to the generator $\xi \in \operatorname{Lie} L G \subset \operatorname{Lie} \hat{L} G$. However, one can check that this condition is not only necessary but also sufficient to define a principal connection $\mathscr{A}$ in $\hat{L} P$.

One can also prove that an arbitrary connection $\mathscr{A}$ in $L P$ can be extended (in many ways) to $\hat{L} P$, i.e., that it is possible to construct a 1 -form $\theta$ on $\hat{L} P$ which satisfies condition (28). The argument is very similar to the one that is used to construct a gauge field in an arbitrary bundle out of local trivializations and a subordinate partition of unity (see, e.g. [21]).

Let $\hat{\mathscr{A}}=(\mathscr{A}, \theta)$ be an extension of $\mathscr{A}$. We will choose $\mathscr{A}$ to be $S^{1}$-equivariant, for example $\mathscr{A}=\bar{A}$. Using the properties in Sect. 4.1 and the fact that $U(1)$ is in the center of $\hat{L} G$, we can easily verify that $\theta$ defines a principal connection in the $U(1)$ bundle $\hat{L} P(L P, U(1))$. Moreover $\mathscr{A}$ vanishes on the $U(1)$ fundamental vectors, and is invariant along the $U(1)$ fibres. Thus it goes to the quotient $\hat{L} P / U(1)$, which is just 
$L P$, and defines a principal connection in $L P(L M, L G)$. Similarly, the curvature, $\Theta$, of the $U(1)$ connection is a basic 2 -form on $\hat{L} P$ and can be viewed as a 2 -form on $L P$. Note that although $d \theta$ is cohomologically trivial on $\hat{L} P, \Theta$ is never trivial as a cohomology class on $L P$. This is because the bundle $\hat{L} P(L P, U(1))$ is always nontrivial, and $\Theta / 2 \pi$ represents its Chern class. In what follows we will show that $\Psi_{2}+\Theta$ is a basic form on the total space of the bundle $L P(L M, L G)$ and that the 2-form on $L M$ which is determined by it is precisely the one that trivializes $\Lambda$.

In $\hat{L} P$ the curvature 2 -form, $\hat{\Omega}$, of $\hat{\mathscr{A}}$ can be decomposed into its $L G$ and $U(1)$ parts

$$
\begin{aligned}
\hat{\Omega}_{U(1)} & =d \theta+\frac{1}{2}[\mathscr{A}, \mathscr{A}]_{U(1)}=\Theta+\frac{1}{2} \omega(\mathscr{A}, \mathscr{A}), \\
\hat{\Omega}_{L G} & =d \mathscr{A}+\frac{1}{2}[\mathscr{A}, \mathscr{A}]_{L G} .
\end{aligned}
$$

As for $d \theta$, all the curvature components in the above equations can be considered as well-defined forms on $L P$.

To prove that the 2 -form $\Psi_{2}+\Theta$ on $L P$ is basic, we must show that for any fundamental vector field $\tilde{\xi}$ with $\xi \in \operatorname{Lie} L G$

$$
\begin{aligned}
i_{\tilde{\xi}}\left(\Psi_{2}+\Theta\right) & =0, \\
\mathscr{L}_{\tilde{\xi}}\left(\Psi_{2}+\Theta\right) & =0 .
\end{aligned}
$$

To verify the first condition we observe that $\Omega_{U(1)}$ vanishes on fundamental vectors of Lie $\hat{L} G$. Thus from (29) $i_{\tilde{\xi}} \Theta=-\omega(\xi, \mathscr{A})$ while, from $(22), i_{\tilde{\xi}} \Psi_{2}=+\omega(\xi, \mathscr{A})$. As for the second condition, using (31) and (27) we have

$$
\mathscr{L}_{\tilde{\xi}}\left(\Psi_{2}+\Theta\right)=\left(i_{\tilde{\xi}} d+d i_{\tilde{\xi}}\right)\left(\Psi_{2}+\Theta\right)=i_{\tilde{\xi}} d \Psi_{2}=-i_{\tilde{\xi}} \pi_{L}^{*}(2 \pi \Lambda)=0 .
$$

This shows that there exists a unique 2 -form $H$ on $L M$ such that

$$
\Psi_{2}+\Theta=-\pi_{L}^{*}(2 \pi H) .
$$

However, since $\Theta$ is closed we also have

$$
\pi_{L}^{*} \Lambda=-\frac{1}{2 \pi} d\left(\Psi_{2}+\Theta\right)=d \pi_{L}^{*} H=\pi_{L}^{*} d H,
$$

which implies that in fact $\Lambda=d H$. This concludes our proof that the vanishing of $\Lambda(=E \lambda)$ in cohomology of $L M$ is a necessary condition for the existence of a string structure.

To prove that the vanishing of $\Lambda=E \lambda$ is also a sufficient condition for the existence of a string structure we will use the explicit construction of $U(1)$ bundles over simply connected spaces which is described in Appendix B. First we show that the vanishing of $\Lambda$ allows one to introduce a closed integral form $\vartheta / 2 \pi$ on $L P$, which when restricted to the fibres is equal to $-\omega / 2 \pi$. Then we construct the total space of $\hat{L} P$ as a $U(1)$ bundle over $L P$ (with $\vartheta / 2 \pi$ being its Chern class). Finally, we exhibit a free $\widehat{L} G$ action on $\widehat{L} P$ and prove that $\widehat{L} P$ is a string structure for $P(M, G)$.

It is in this part of the proof that we need to assume that $M$ is 2-connected. From the homotopy exact sequence for $P(M, G)$ we then find $\pi_{i}(P)=0, i=0,1,2$ (see, Example 2 in Appendix A). Then for the corresponding loop bundle we have 
$\pi_{i}(L P)=0, i=0,1$. Thus any curvature 2 -form of a $U(1)$-bundle over $L P$ determines this bundle completely.

From the discussion in Sect. 2 we know that if $M$ is 2-connected then $E$ is an injection from $H^{4}(M, \mathbf{R})$ into $H^{3}(L M, \mathbf{R})$. Thus, from the vanishing of $E \lambda$ in the cohomology of $L M$ we deduce that there exists a 3 -form $h$ on $M$ such that $\lambda=d h$. Let $\mathscr{A}=\bar{A}$ and $\Psi_{2}$ be the corresponding Chern-Simons form on LP. Then

$$
\vartheta=-\Psi_{2}+\pi_{L}^{*} E(2 \pi h)
$$

is a closed 2-form on $L P$ which, on the fibres, is equal to - $\omega$. Comparing with (33) it is clear that $\vartheta$ looks very much like $\Theta$. However, in order to prove that $\vartheta$ can be considered as a curvature form of some $U(1)$-bundle we still need to show that $\vartheta / 2 \pi$ represents an integral cohomology class in $L P$.

To prove that $\vartheta / 2 \pi$ is integral we observe that it can also be written as

$$
\frac{1}{2 \pi} \vartheta=-E\left(\omega_{3}-\pi^{*} h\right)-d r
$$

where $Y$ is given by (23). On the other hand the 3 -form $h$ on $M$ is only determined up to the addition of an exact form. It is standard in the theory of Chern-Simons forms [20] that for $\lambda=d h$ one can use this freedom to adjust $h$ such that the closed 3 -form $\omega_{3}-\pi^{*} h$ represents an integral cohomology class in $L P$. The image of this form by $E$ is also an integral form, which by (34) is cohomologous to $-\vartheta / 2 \pi$.

We can now use the construction from Appendix B and apply it to $L P$ with the closed, integral form $\vartheta / 2 \pi$. The total space of the resulting $U(1)$-bundle $\hat{L} P$ can be represented by equivalence classes of triples $\left[p, l_{\left(p_{0}, p\right)}, \alpha\right]$, where $p \in L P, l_{\left(p_{0}, p\right)}$ is a path in $L P$ from a fixed base point $p_{0}$ to $p$ and $\alpha \in U(1)$. The precise form of the equivalence relation is given in Appendix B.

A similar method was used in Sect. 3 to construct the central extension of the loop group. We can now put both of them together and show that $\hat{L} P$ is in fact an $\hat{L} G$ bundle. Let $\hat{p}=\left[p, l_{\left(p_{0}, p\right)}, \alpha\right]$ and $\hat{g}=[g, l(g), u]$ represent points in $\hat{L} P$ and $\hat{L} G$, respectively. Consider the following expression:

$$
\left[p, l_{\left(p_{0}, p\right)}, \alpha\right] \cdot[g, l(g), u]=\left[p g, l_{\left(p_{0}, p\right)} * p l(g), \alpha u\right],
$$

where $p l(g)$ is a path in the fibre of $L P$ from $p$ to $p g$. It is not difficult to convince oneself that the equivalence class of the element on the right-hand side depends only on the equivalence classes of the factors on the left-hand side and thus (35) defines an action of $\hat{L} G$ on $\hat{L} P$. In fact this action is free and gives to $\hat{L} P$ a structure of a $\hat{L} G$-bundle over $L M$. In this bundle we have following projections:

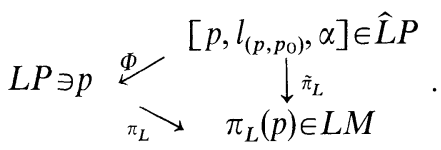

From this diagram it is almost obvious that $\hat{L} P$ together with the projection $\Phi$ define a string structure. This completes the proof that the vanishing of $E \lambda$ is also a sufficient condition for the existence of a string structure.

4.4. Nonequivalent String Structures. A natural question that arises is to what 
extent a string structure $\hat{L} P$ is determined by $L P$ and $\hat{L} G$. If we think about $\widehat{L} P$ as a $U(1)$-bundle over $L P$, then the choice of the central extension $\hat{L} G$ fixes this bundle over the fibres of $L P$; however, there remains a freedom in extending this bundle from the fibres to the total space $L P$. One can easily enumerate all nonequivalent extensions. Let $\hat{L} P_{(1)}$ and $\hat{L} P_{(2)}$ be two string structures with the corresponding $U(1)$ curvatures $\Theta_{(1)}$ and $\Theta_{(2)}$, respectively. By subtracting Eqs. (31) and (32) with $\Theta=\Theta_{(1)}$ from the same equations with $\Theta=\Theta_{(2)}$, we obtain

$$
\begin{array}{r}
i_{\widetilde{\xi}}\left(\Theta_{(1)}-\Theta_{(2)}\right)=0 \\
\mathscr{L}_{\tilde{\xi}}\left(\Theta_{(1)}-\Theta_{(2)}\right)=0
\end{array} \quad \xi \in \operatorname{Lie} L G
$$

which shows that $\Theta_{(1)}-\Theta_{(2)}$ is a basic form on $L P$. Thus

$$
\Theta_{(1)}=\Theta_{(2)}+\pi_{L}^{*} H_{12},
$$

where $H_{12}$ is a 2-form on $L M$. Since $\Theta_{(1)}$ and $\Theta_{(2)}$ are closed and integral forms on $L P, H_{12}$ is a closed and integral form on $L M$, i.e., it defines an element in $H^{2}(L M, \mathbf{Z})$. Before we conclude that nonequivalent string structures are parametrized by $H^{2}(L M, \mathbf{Z})$, we must check that $\pi_{L}^{*}: H^{2}(L M, \mathbf{Z}) \rightarrow H^{2}(L P, \mathbf{Z})$ is an injection. When $M$ is 2-connected this can be deduced as follows:

(i) from the long homotopy exact sequence for the fibration $L P(L M, L G)$ we find that $\left(\pi_{L}\right)_{*}: \pi_{2}(L P) \rightarrow \pi_{2}(L M)$ is a surjection (because $\pi_{1}(L G)=0$ );

(ii) from the Hurewicz theorem (i) implies that $\left(\pi_{L}\right)_{*}$ is a surjection from $\mathrm{H}_{2}(L P, \mathbf{Z})$ onto $\mathrm{H}_{2}(L M, \mathbf{Z})$;

(iii) by duality $\pi_{L}^{*}: H^{2}(L M, \mathbf{Z}) \rightarrow H^{2}(L P, \mathbf{Z})$ is an injection. Therefore nonequivalent string structures are parametrized by $H^{2}(L M ; \mathbf{Z})$.

This concludes our discussion of the existence of string structures in the free case.

4.5. Generalizations. The above discussion can be straightforwardly generalized to the case where $G=G_{1} \times \cdots \times G_{n}$. Let $\omega=\sum_{i} m_{i} \omega^{(i)}, m_{i} \in \mathbf{Z}$, be the cocycle that defines $\hat{L} G$. A gauge field in $L P$ can be decomposed into components $\mathscr{A}^{(i)}$, $i=1, \ldots, n$, where $\mathscr{A}^{(i)}$ is the projection of $\mathscr{A}$ onto Lie $L G_{i}$. Let $\Psi_{2}^{(i)}, i=1, \ldots, n$, be 2 -forms obtained by substituting in (22) $\bar{A}$ with $\mathscr{A}^{(i)}$. Then the only modification which is needed to extend the previous discussion to this more general case is to substitute everywhere $\Psi_{2}$ by $\sum_{i} m_{i} \Psi_{2}^{(i)}$. The result is that the necessary and sufficient condition for the existence of a string structure corresponding to the cocycle $\omega$ is

$$
\sum_{i=1}^{n} m_{i} E \lambda^{(i)}=0 \quad \text { in } \quad H^{3}(L M, \mathbf{R})
$$

\section{Existence of String Structures and Obstruction Theory}

5.1. Extension of the Structure Group of a Principal Fibre Bundle. Our discussion here will closely follow ref. [22]. Let $(\hat{H}, \varphi)$ be an extension of the 
group $H$ in the sense of Sect. 3.2 and $P(X, H)$ a principal $H$-bundle over some space $X$. We say that this bundle admits a $\hat{H}$ extension if there exists a principal bundle $\hat{P}(X, \hat{H})$ and a bundle map $\Phi: \hat{P} \rightarrow P$ such that $\Phi(\hat{p} \hat{h})=\Phi(\hat{p}) \varphi(\hat{h})$.

The existence of such extensions can be studied using the classification theorem for principal fibre bundles [23]. This theorem says that for any group $G$ there exists a universal principal $G$-bundle $E_{G}\left(B_{G}, G\right)$ whose total space $E_{G}$ is contractible. Its base $B_{G}$ is then called a classifying space for principal $G$-bundles and enjoys the following property: any principal $G$-bundle $P$ is obtained as a pullback of $f_{P}^{*}\left(E_{G}\right)$, where $f_{P}$ is a map from $X$ to $B_{G}$. The map $f_{P}$ is called the classifying map of the bundle $P$. Moreover, any two maps $f_{P}$ and $f_{P}^{\prime}$ which are homotopic lead to isomorphic bundles $P, P^{\prime}$ and vice-versa.

5.1.1. Relation Between $B_{H}$ and $B_{\hat{H}}$. For a given group the classifying space is determined only up to homotopy equivalence. In the case we want to study this gives us the freedom of choosing the classifying spaces $B_{\hat{H}}$ and $B_{H}$ such that they form a fibration

$$
B_{N} \rightarrow B_{\hat{H}}
$$

whose fibre is the classifying space of $N=\operatorname{ker} \varphi$. Since this property is less known we recall briefly its proof.

The first observation is that since $N \subset \hat{H}$, it acts freely on $E_{\hat{H}}$, and we may therefore consider the quotient $E_{\hat{H}} / N$. Thus $E_{\hat{H}}$ is also a principal $N$-bundle over $E_{\hat{H}} / N$ and, since $E_{\hat{H}}$ is contractible by hypothesis, $B_{N}=E_{\hat{H}} / N$ is a classifying space for $N$-principal bundles. The second observation is that $B_{N}$ is a $H$-principal bundle over $B_{\hat{H}}$. Indeed, starting with $E_{\hat{H}}$, we may build the associated bundle $E_{\hat{H}} \times{ }_{\hat{H}} \hat{H} / N$, which is a $\hat{H} / N$-bundle over $B_{\hat{H}}$. Since this associated bundle is nothing else but $E_{\hat{H}} / N$ itself, the conclusion stems from the fact that $H$ is isomorphic with $\hat{H} / N$. The third observation is that we can build a space $B_{\hat{H}}^{\prime}$ which has the same homotopy type as $B_{\hat{H}}$ and which can be fibrated over $B_{H}$ with fibres $B_{N}$. We construct it as the bundle $B_{\hat{H}}^{\prime}=E_{H} \times{ }_{H} B_{N}$, associated with $E_{H}\left(B_{H}, H\right)$ by using the $H$-action on $B_{N}\left(B_{\hat{H}}, H\right)$. The fact that $B_{\hat{H}}^{\prime}$ has the same homotopy type as $B_{\hat{H}}$ can be seen formally by noticing that, locally,

$$
B_{\hat{H}}^{\prime} \simeq B_{N} \times B_{H} \simeq E_{\hat{H}} / N \times E_{H} / H \simeq E_{\hat{H}} / \hat{H} \times E_{H} \simeq B_{\hat{H}} \times E_{H},
$$

where $E_{H}$ is contractible. Since classification of principal bundles is a homotopy classification [23], we can forget the difference between $B_{\hat{H}}$ and $B_{\hat{H}}^{\prime}$ and use the latter as a classifying space for $\hat{H}$-principal bundles (from now on we will write $B_{\hat{H}}$ rather than $B_{\hat{H}}^{\prime}$.

The only thing to remember is that whenever we have an exact sequence

$$
0 \rightarrow N \rightarrow \hat{H} \stackrel{\varphi}{\longrightarrow} H \rightarrow 0,
$$

we may both consider $\hat{H}$ as a principal $N$-bundle over $H$ and $B_{\hat{H}}$ as a $B_{N}$-bundle over $B_{H}$ (of course, the latter is not a principal bundle!). We will denote by $\rho$ the projection $B_{\hat{H}} \rightarrow B_{H}$. 
5.1.2. Condition for the Extension of the Structure Group. In order to extend $P(X, H)$ to $\hat{P}(X, \hat{H})$, it is necessary and sufficient to find a classifying map $f_{\hat{P}}$ from $X$ to $B_{\hat{H}}$ making the following diagram commutative:

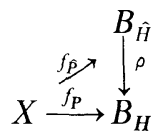

Such a map $f_{\hat{P}}$ can be considered as a section of the bundle $f_{P}^{*}\left(B_{\hat{H}}\right)$, with base $X$ and fibre $B_{N}$ obtained by pulling back $B_{\hat{H}}\left(B_{H}, B_{N}\right)$ along $f_{P}$.

Finding a global section in a given bundle with base $X$ and fibre $F$ is of course not always possible, and this is the subject of the obstruction theory [24]. The idea is to build the section explicitly by using a simplicial decomposition of $X$ : there is no problem at the level of 0 -simplices but when $\pi_{0}(F) \neq 0$, there is a potential problem at the level of 1-simplices; one can prove that the obstruction lies in $H^{1}\left(X, \pi_{0}(F)\right)$. If a lift of the 1-skeleton can be constructed, one tries to extend it to the 2 -skeleton, and so on. In general, in order to extend the section from the $n$-skeleton to the $(n+1)$-skeleton, one finds an obstruction in $H^{n}\left(X, \pi_{n-1}(F)\right)$.

In the present case $F=B_{N}$, and we find a sequence of obstructions given by classes in $H^{n}\left(X, \pi_{n-1}\left(B_{N}\right)\right)$.

5.1.3. Example: Spin Structures. As a particular example of these techniques, let us consider the case where $H=S O(n), \hat{H}=\operatorname{Spin}(n)$ and $N=\mathbf{Z}_{2}$. Using $\pi_{k}\left(B_{N}\right)=$ $\pi_{k-1}(N)$ we find that the only nontrivial homotopy group of $B_{\mathbf{Z}_{2}}$ is $\pi_{1}\left(B_{\mathrm{Z}_{2}}\right)=$ $\pi_{0}\left(\mathbf{Z}_{2}\right)=\mathbf{Z}_{2}$. The only possible obstruction is therefore an element $w_{2}$ in $H^{2}\left(M, \mathbf{Z}_{2}\right)$, and we recover the usual condition for the existence of spin structures.

5.2. Obstruction to String Structures. We now apply the preceding techniques to the case of $U(1)$-extensions of principal loop bundles. Then $N, \hat{H}$ and $H$ are replaced by $U(1), \hat{L} G$ and $L G$ respectively. The possible obstructions lie a priori in $H^{n}\left(L M, \pi_{n-1}\left(B_{U(1)}\right)\right)$. Since $\pi_{n-1}(B U(1))=\pi_{n-2}(U(1))$ and the only nontrivial homotopy group of $S^{1}$ is $\pi_{1}(U(1))=\mathbf{Z}$, we see that the only possible obstruction for a string structure is a particular class in $H^{3}(L M, \mathbf{Z})$.

We must now identify this obstruction explicitly. Actually this has already been done, at least for some cases, in Sect. 4. We remind the reader that we introduced a map $E$ (the composition of evaluation map and integration over the fibre $S^{1}$ ) going from $H^{k}(M)$ to $H^{k-1}(L M)$. We saw that the obstruction could be written as $E \lambda$. We will see that, in general, $\lambda$ is an element of $H^{4}(M, \mathbf{Z})$ and is actually a characteristic class since it comes from $H^{4}\left(B_{G}, \mathbf{Z}\right)$ via the classifying map. Indeed we have the following picture:

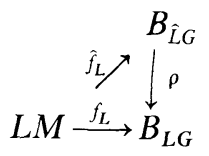

where $f_{L}$ is the classifying map of $L P(L M, L G)$ and $\hat{f}_{L}$ the classifying map of 
$\hat{L} P(L M, \hat{L} G)$. We know (cf. A.5) that $H^{3}\left(B_{L G}, \mathbf{Z}\right)=\mathbf{Z}$ and $H^{3}\left(B_{\hat{L} G}, \mathbf{Z}\right)=0$; therefore the obstruction is the characteristic class obtained by pulling back $\left(v i a f_{L}\right)$ the generator of the third cohomology of $B_{L G}$. One can convince oneself that $B_{L G}$ can be identified with the space of loops $L B_{G}$ of $B_{G}$, but $H^{3}\left(L B_{G}\right) \simeq H^{4}\left(B_{G}\right)$, a property which can be proved by using the evaluation map $S^{1} \times L B_{G} \rightarrow B_{G}$ and integrating over the fibre (notice that $H^{4}\left(B_{G}\right)$ is the smallest cohomology group of $B_{G}$ when $G$ is semi-simple since $H^{4}\left(B_{G}\right) \simeq H^{3}(G)$ ). We have the following diagram

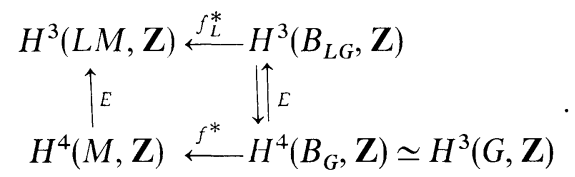

The obstruction can therefore be written as $E \lambda \in H^{3}(L M, \mathbf{Z})$, where $\lambda \in H^{4}(M, \mathbf{Z})$ is a characteristic class.

In the case where $P$ is the spin bundle, $\lambda$ is actually the integer class $\frac{1}{2} p_{1}$, where $p_{1}$ is the Pontrjagin class of the tangent bundle.

Notice that $E \lambda=0$ does not necessarily imply $\lambda=0$. We discussed this already in Sect. 2.

Finally, we would like to remark that there is actually a faster-but more abstract-way to arrive at the same conclusion. This method was used in [1]. The idea is to consider the exact sequence

$$
0 \longrightarrow U(1) \stackrel{\iota}{\longrightarrow} \hat{L} G \stackrel{\varphi}{\longrightarrow} L G \longrightarrow 0
$$

and the associated cohomology sequence

$$
\left.\longrightarrow H^{1}(L M, U(1)) \stackrel{i_{*}}{\longrightarrow} H^{1}(L M, \hat{L} G)\right) \stackrel{\varphi_{*}}{\longrightarrow} H^{1}(L M, L G) \stackrel{\delta_{*}}{\longrightarrow} H^{2}(L M, U(1)) .
$$

It happens that such a sequence is meaningful, at least in the lowest degrees, although $L G$ and $\hat{L} G$ are not abelian [25]. Moreover, the bundles $L P$ and $\hat{L} P$ can be considered as elements of $H^{1}(L M, L G)$ and $H^{1}(L M, \hat{L} G)$, respectively. Therefore,

$$
\left[\exists \hat{L} P \in H^{1}(L M, \hat{L} G) \quad \text { such that } \quad \varphi_{*}(\hat{L} P)=L P\right] \Leftrightarrow\left[L P \in \operatorname{ker} \delta_{*}\right],
$$

i.e., $\delta_{*}(L P)=H^{2}(L M, U(1))=0$. Hence the obstruction is equal to $\delta_{*}(L P)$ and lies in $H^{2}(L M, U(1))$, which, by the universal coefficient theorem [21], can be related to $H^{3}(L M, \mathbf{Z})$. The same homological argument tells us that if $\hat{L} P_{1}$ and $\hat{L} P_{2}$ are two nonequivalent lifts, we obtain $\varphi_{*}\left(\hat{L} P_{1}\right)=\varphi_{*}\left(\hat{L} P_{2}\right)$ yielding $\varphi_{*}\left(\hat{L} P_{1}-\hat{L} P_{2}\right)=0$, i.e., $\hat{L} P_{1}-\hat{L} P_{2} \in i_{*} H^{1}(L M, U(1))$. Then $\hat{L} P_{2}=\hat{L} P_{1}+i_{*}(\beta)$, where $\beta \in H^{1}(L M, U(1))$, and we find that nonequivalent lifts are parametrized by elements of $H^{1}(L M, U(1))$. This, in turn, can be related to $H^{2}(L M, \mathbf{Z})$, cf. Sect. 4.4.

\section{Examples}

6.1. String Structures on Coset Spaces $G / H$. Let us assume for simplicity that both $G$ and $H$ are simply connected, and that $G$ is simple while $H=H_{1} \times \cdots \times H_{n}$ is, 
in general, a product of $n$ simple factors.

On any coset space $G / H$ we have a canonical $H$-bundle

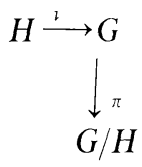

whose total space is the group $G$ itself. The corresponding loop bundle is

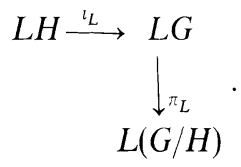

Let $\widehat{L} G$ be the basic central extension of $L G$. The induced bundle $i_{L}^{*} \hat{L} G$ over $L H$ defines a central extension of $L H$ associated with the cocycle.

$$
\omega^{G}=\sum_{i=1}^{n} m_{i} \omega^{H_{i}}
$$

where $\omega^{H_{\imath}}$ is the basic cocycle on $L H_{i}$ and $m_{i} \in \mathbf{Z}$ is the embedding index ${ }^{2}$ of $H_{i}$ in $G$. It is obvious that for this particular central extension of $L H, \hat{L} G$ defines a corresponding string structure in the bundle $L G(L(G / H), L H)$.

With our assumptions $G / H$, is a 2-connected space and therefore always a spin manifold. We can also consider the existence of a string structure for the spin bundle $S(G / H, \operatorname{Spin}(d)), d=\operatorname{dim}(G)-\operatorname{dim}(H)$. This bundle reduces to the canonical $H$-bundle $G$ and we have

$$
S(G / H, \operatorname{Spin}(d)) \simeq G(G / H, H) \times{ }_{H} \operatorname{Spin}(d),
$$

where the right-hand side consists of equivalence classes $\left[\left(g h, \rho\left(h^{-1}\right) s\right)\right], g \in G$, $s \in \operatorname{Spin}(d), h \in H$ and $\rho: H \rightarrow \operatorname{Spin}(d)$ is the embedding via the isotropy representation of $H$. The corresponding loop bundle is isomorphic to $L G(L(G / H), L H) \times_{L H}$ $L$ Spin $(d)$, where we use the embedding $\rho_{L}: L H \rightarrow L \operatorname{Spin}(d)$ induced by $\rho$. Note also that the total space of the bundle $L G$ is a subspace of the total space of the loop bundle $L S$. Thus a string structure (if it exists) in the loop bundle $L S$ corresponding to the central extension $\hat{L} \operatorname{Spin}(d)$ induces a $U(1)$-bundle over the total space of the bundle $L G$ and its Chern-class (up to a factor of $2 \pi$ ) restricted to the fibre $L H$ is represented by the cocycle $\omega^{\rho}$ on Lie $L H$ given by

$$
\omega^{\rho}=\sum_{i=1}^{n} m_{i}^{\rho} \omega^{H_{i}},
$$

where $m_{i}^{\rho}$ are the indices of $H_{i}$ in Spin $(d)$ by the embedding $\rho$.

Since $G$ is simple, $H^{2}(L G, \mathbf{Z})$ is one-dimensional. Therefore, even though the space of nonequivalent cocycles on Lie $L H$ is $n$-dimensional, there is only a 1 dimensional subspace of those which correspond to $U(1)$-bundles over $L H$ that

\footnotetext{
2 The embedding index is $m_{i}=\left(n_{H_{l}} / n_{G}\right)\left(c_{1}(\operatorname{Ad} G) / c_{1}\left(\operatorname{Ad} H_{t}\right)\right)$, where $n_{G}$ and $n_{H_{1}}$ are the dual Coxeter numbers of $G$ and $H_{\imath}$, respectively, and $c_{1}(\mathbf{r})$ is the second index of the representation $\mathbf{r}$ of $H_{l}$ defined by $\operatorname{Tr}\left(t_{a} t_{b}\right)=-c_{1}(\mathbf{r}) \delta_{a b}$, with $t_{a}$ being the generators of $H_{i}$ in the representation $\mathbf{r}$
} 
can be extended over $L G$. Using this observation we can deduce that the spin bundle on $G / H$ has a string structure if and only if the cocycles $\omega^{G}$ and $\omega^{\rho}$ on $L H$ satisfy $\omega^{\rho}=m \omega^{G}$, where $m$ is some integer.

Remark. Since $G / H$ is 2-connected, we find that the above relation is a condition for the Pontrjagin class of $G / H$ to vanish.

\section{Example 1. Spheres $S^{n}$}

Since $S^{n}=\operatorname{Spin}(n+1) / \operatorname{Spin}(n)$, the canonical and the spin bundle coincide and there is no problem with defining a string structure. This of course agrees with the well-known fact that $p_{1}\left(S^{n}\right)=0$.

Example 2. Quaternionic projective spaces $H P^{n}$

Quaternionic projective space $H P^{n}=\mathrm{Sp}(n+1) / \mathrm{Sp}(1) \times \mathrm{Sp}(n)$ is a $4 n$-dimensional spin manifold. For the canonical $\operatorname{Sp}(1) \times \operatorname{Sp}(n)$ bundle we find $\omega^{\mathrm{Sp}(n+1)}=$ $\omega^{\mathrm{Sp}(1)}+\omega^{\mathrm{Sp}(n)}$, while for the spin bundle we get $\omega^{\rho}=n \omega^{\mathrm{Sp}(1)}+\omega^{\mathrm{Sp}(n)}$, which shows that $\omega^{\rho}$ is proportional to $\omega^{\mathrm{Sp}(n+1)}$ only for $n=1$ and this is the only case where one can define a string structure for the spin bundle of $H P^{n}$. Once more this agrees with $p_{1}\left(H P^{n}\right) \neq 0, n \geqq 2$ [26], while, for $n=1, H P^{1}=S^{4}$, and this case reduces to the previous example. Notice, however, that $\mathrm{Sp}(n+1)$, considered as a principal bundle over $H P^{n}$ always admits a string structure.

Remark. Real projective spaces $R P^{n}$ are not simply connected. On the other hand complex projective spaces $C P^{n}$ admit a spin structure only for $n$ odd. Since the total Pontrjagin class is $p\left(C P^{n}\right)=(1-x)^{n+1}$, where $x$ is a generator in dimension $2, p_{1}\left(C P^{n}\right) \neq 0$ except for $n=1$. However, $C P^{1}=S^{2}$, so this example is rather trivial.

6.2. String Structures in Hopf Bundles. Consider SU(2)-principal bundles of the form

$$
S U(2) \rightarrow \underset{\downarrow}{\downarrow P^{n}} \quad n \geqq 1 .
$$

They correspond to the nontrivial $S U(2)$-instanton on $H P^{n}$ with $k=1$, and it is well-known that the second Chern-class is nontrivial. Thus these bundles cannot admit a string structure. This can also be checked explicitly as follows. The total space of the corresponding loop bundle, $L S^{4 n+3}, n \geqq 1$, is 2 -connected which implies that any $U(1)$-bundle over it is trivial. This excludes the existence of a string structure since it would lead to a trivial $U(1)$-bundle over the fibres $L S U(2)$ (cf. Remark 3 in Sect. 3.5).

\section{Appendix A. Homotopy Groups of Loop Spaces and Loop Groups}

A.1. Long Exact Homotopy Sequence of a Fibration. The basic tool in computing the homotopy groups of a fibration

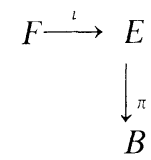


is the long exact sequence of homotopy groups [21],

$$
\begin{gathered}
\cdots \rightarrow \pi_{k}(F) \stackrel{i_{*}}{\longrightarrow} \pi_{k}(E) \stackrel{\pi_{*}}{\longrightarrow} \pi_{k}(B) \stackrel{\delta_{*}}{\longrightarrow} \pi_{k-1}(F) \rightarrow \cdots \\
\cdots \rightarrow \pi_{0}(F) \rightarrow \pi_{0}(E) \rightarrow \pi_{0}(B) \rightarrow 0 .
\end{gathered}
$$

Here $i_{*}$ and $\pi_{*}$ are the homomorphisms between homotopy groups induced, respectively, by the inclusion of the fibre $F$ in the total space $E$ and the projection onto the base $M$. The extent to which the fibration is not a product is measured by the homomorphism $\delta_{*}$ called the "homotopy-holonomy" operator.

Example 1. Universal $G$-bundle $E_{G}\left(B_{G}, G\right)$.

Since $E_{G}$ is contractible, all the homotopy groups of $E_{G}$ vanish. Then one obtains the well known relations

$$
\pi_{k}(G)=\pi_{k+1}(B G)
$$

Example 2. $P(M, G)$ a principal bundle, and $G$ a simply connected Lie group.

Since $G$ is a Lie group, we must have $\pi_{i}(G)=0, i \leqq 2$, and the long exact homotopy sequence implies that

$$
\pi_{i}(M)=\pi_{i}(P), \quad i=0,1,2 .
$$

In particular, if $M$ is 2-connected, the total space $P$ is 2-connected as well.

A.2. Homotopy Groups of a Loop Space. The loop space $L X$ can be viewed as a fibration

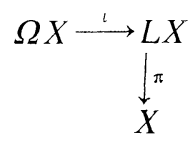

where $\Omega X$ is the space of based loops at $x_{0} \in X, i$ is the natural inclusion and $\pi$ is the projection onto the origin of the loop. It is a standard fact ${ }^{3}$ in topology that $\pi_{k}(\Omega X)=\pi_{k+1}(X)$. Since any closed sphere in $X$ can be lifted to $L X$, for example as a sphere of constant loops, the $\delta_{*}$ map is trivial. Therefore the homotopy sequence can be truncated after each $\delta_{*}$, so that we get

$$
\underset{\|}{\stackrel{\delta_{*}}{\longrightarrow} \pi_{k}(\Omega X) \stackrel{i_{*}}{\longrightarrow} \pi_{k}(L X) \stackrel{\pi_{*}}{\longrightarrow} \pi_{k}(X) \stackrel{\delta_{*}}{\longrightarrow} 0} \underset{\pi_{k+1}(X)}{\|}
$$

where $i_{*}$ is one to one while $\pi_{*}$ is onto. Consequently,

$$
\pi_{k}(L X) / \pi_{k}(X)=\pi_{k+1}(X) .
$$

In particular, if $X$ is 2 -connected, $L X$ is simply connected.

\footnotetext{
${ }^{3}$ As a matter of fact this can also be shown by considering the homotopy sequence associated with the fibration of the space, $P X$, of paths in $X$ with fibre $\Omega X$ and base $X$
} 
A.3. Homotopy Groups of $L G$. Since the loop group $L G$ is topologically a product $L G \simeq G \times \Omega G$, the homotopy groups factorize [21],

$$
\pi_{k}(L G)=\pi_{k}(G) \times \pi_{k}(\Omega G)=\pi_{k}(G) \times \pi_{k+1}(G) .
$$

If $G$ is a simply connected simple Lie group, this implies $\pi_{0}(L G)=\pi_{1}(L G)=0$ and $\pi_{2}(L G)=\pi_{3}(G)=Z$.

A.4. Homotopy Groups of $\hat{L} G$. Consider the case where $G$ is simple and simply connected. Since $\hat{L} G$ is a $U(1)$-bundle over $L G$ and $\pi_{k}(U(1))=0, k>1$, we must have

$$
\pi_{k}(\hat{L} G)=\pi_{k}(L G), \quad k \geqq 3 .
$$

For $k \leqq 2$ the homotopy sequence becomes

$$
\begin{array}{r}
0 \rightarrow \pi_{2}(\hat{L} G) \rightarrow \pi_{2}(L G) \stackrel{\delta_{*}}{\rightarrow} \pi_{1}(U(1)) \rightarrow \pi_{1}(\hat{L} G) \rightarrow 0 . \\
\mathbb{Z}
\end{array}
$$

Here $\delta_{*}$ is given by evaluating the Chern class of $\hat{L} G$ on the generator of $\pi_{2}(L G)$. If $\hat{L} G$ is the basic central extension, $\delta_{*}$ is an isomorphism and we get $\pi_{1}(\hat{L} G)$ $=\pi_{2}(\hat{L} G)=0$. For higher extensions associated with cocycles $m \omega, m \in \mathbf{Z}$, we find that $\pi_{1}(\hat{L} G)=\mathbf{Z} / m \mathbf{Z}$ and $\pi_{2}(\hat{L} G)=0$.

A.5. Homotopy Groups and (co)homology Groups of $B_{G}, B_{L G}$ and $B_{\hat{L} G}$. Let $G$ simple and simply connected, and $\hat{L} G$ the basic central extension. Then, from A.1 and A.4, we have

$$
\begin{gathered}
\pi_{i}\left(B_{G}\right)=0 \quad i \leqq 3, \quad \pi_{4}\left(B_{G}\right)=\mathbf{Z}, \\
\pi_{i}\left(B_{L G}\right)=0 \quad i \leqq 2, \quad \pi_{3}\left(B_{L G}\right)=\mathbf{Z}, \\
\pi_{i}\left(B_{\hat{L} G}\right)=0, \quad i \leqq 3 .
\end{gathered}
$$

The Hurewicz theorem for a $q$-connected space $X(q \geqq 1)$ reads

$$
\pi_{i}(X)=H_{i}(X, \mathbf{Z}) \text { for } i \leqq q+1 .
$$

On the other hand the universal coefficient theorem gives

$$
H^{i}(X, \mathbf{Z}) \simeq H_{i}(X, \mathbf{R}) \oplus T_{i-1}(X),
$$

where $T_{i-1}(X)$ is the torsion part of $H^{i}(X, \mathbf{Z})$. Henceforth we find

$$
\begin{array}{rl}
H^{i}\left(B_{G}, \mathbf{Z}\right)=0, & i \leqq 3, \quad H^{4}\left(B_{G}, \mathbf{Z}\right)=\mathbf{Z}, \\
H^{i}\left(B_{L G}, \mathbf{Z}\right)=0, & i \leqq 2, \quad H^{3}\left(B_{L G}, \mathbf{Z}\right)=\mathbf{Z}, \\
H^{i}\left(B_{\hat{L} G}, \mathbf{Z}\right)=0 & i \leqq 3 .
\end{array}
$$

\section{Appendix B. $U(1)$-Bundles on Simply Connected Spaces}

On a simply connected space $X$ a closed 2-form $\omega$, such that $\omega / 2 \pi$ represents an integer cohomology class in $H^{2}(X, \mathbf{R})$, defines, up to an isomorphism, a principal $U(1)$ fibre bundle $P$ over $X$. Reciprocally, such a $U(1)$-bundle determines the 
cohomology class of $\omega$. Although this property is well-known, we prefer to recall its proof here, since some of the ingredients entering the derivation play an important role in Sect. 4.

In order to establish the above correspondence, we first fix a base point $x_{0} \in X$ and consider the set of triples

$$
\mathscr{P}=\left\{\left[x, l_{\left(x_{0}, x\right)}, \alpha\right] ; x_{0} \in X, l_{\left(x_{0}, x\right)} \text { a path in } X \text { from } x_{0} \text { to } x, \alpha \in U(1)\right\}
$$

along with the equivalence relation

$$
[x, l(\tau), \alpha] \sim\left[x^{\prime}, l^{\prime}(\tau), \alpha^{\prime}\right] \quad \text { iff } \quad x=x^{\prime} \quad \text { and } \quad \alpha^{\prime}=\Delta\left(l^{\prime} * l^{-1}\right) \alpha,
$$

where

$$
\Delta\left(l^{\prime} * l^{-1}\right)=e^{-i \int_{\sigma\left(l^{\prime} * l^{-1}\right)^{\omega}},}
$$

where $\sigma\left(l^{\prime} * l^{-1}\right)$ is any surface in $X$ whose boundary is a closed loop $l^{\prime} * l^{-1}$ obtained by going first along path $l^{\prime}$ and then back along $l$.

Remark. The integrality condition for $\omega / 2 \pi$ implies that the phase $\Delta\left(l^{\prime} * l^{-1}\right)$ does not depend on the choice of $\sigma\left(l^{\prime} * l^{-1}\right)$. Such a surface always exists since $X$ is simply connected.

To show that $\mathscr{P} / \sim$ is a $U(1)$-bundle, we exhibit a projection $\pi$ onto the base and a free $U(1)$ action along the fibres. They are defined as follows:

$$
\begin{gathered}
\pi\left(\left[x, l_{\left(x_{0}, x\right)}, \alpha\right]\right)=x, \\
R_{\beta}\left(\left[x, l_{\left(x_{0}, x\right)}, \alpha\right]\right)=\left[x, l_{\left(x_{0}, x\right)}, \alpha \beta\right], \quad \beta \text { in } U(1) .
\end{gathered}
$$

It is obvious that these definitions do not depend on the representative of an equivalence class in $\mathscr{P}$. By choosing a local cover in the space of paths in $X$ that originate at $x_{0}$ one can also exhibit an explicit local trivialization of $\mathscr{P} / \sim$.

Finally we describe a connection $\theta$ in $\mathscr{P} / \sim$ whose curvature is equal to $\omega$. The easiest way to define such a $\theta$ is to give an explicit prescription for the parallel transport in $\mathscr{P} / \sim$. Let $l_{\left(x, x^{\prime}\right)}$ be a path in $X$ and $\left[x, l_{\left(x_{0}, x\right)}, \alpha\right]$ a point in $\mathscr{P} / \sim$ which we want to parallely transport along $l_{\left(x, x^{\prime}\right)}$. The result is $\left[x^{\prime}, l_{\left(x, x^{\prime}\right)} * l_{\left(x_{0}, x\right)}, \alpha\right]$, where the path $l_{\left(x, x^{\prime}\right)} * l_{\left(x_{0}, x\right)}$ from $x_{0}$ to $x^{\prime}$ is obtained by the usual composition of the path $l_{\left(x_{0}, x\right)}$ with $l_{\left(x, x^{\prime}\right)}$. Once more one can check that this does not depend on the representative in the equivalence class and defines the correct connection $\theta$.

\section{Remarks.}

1. This representation of line bundles using equivalence classes of paths and phases is particularly convenient if we want to study explicit lifts of the action of some symmetry group, $S$, on the base, to the total space of the bundle. In the present case $S$ is Diff $S^{1}$ and its action has the following properties: (1) the point $x_{0}$ is a fixed point of the $S$ action, (2) $\omega$ is $S$ invariant. In such a situation an explicit lift is given by

$$
s\left[x, l_{\left(x_{0}, x\right)}, \alpha\right]=\left[s x, s l_{\left(x_{0}, x\right)}, \alpha\right], \quad s \in S,
$$

where $s l_{\left(x_{0}, x\right)}(\tau)=s\left(l_{\left(x_{0}, x\right)}(\tau)\right)$ is the image of the path $l_{\left(x_{0}, x\right)}$ under $s$.

2. The previous construction of the $U(1)$ bundle can also be described as follows. Let $P_{x_{0}} X$ be the space of based paths in $X$, i.e., the space of paths $l:[0,1] \rightarrow X$ with origin $x_{0}, l(0)=x_{0}$. Let $\Omega_{x_{0}} X$ be the space of based loops in $X$ which carries a natural group 
structure. Then, the map $\pi: P_{x_{0}} X \rightarrow X$ associating a path with its endpoint is a bundle projection. Indeed, if $l$ and $l^{\prime}$ are two paths with the same endpoint, then $l^{\prime} * l^{-1}$ is a closed loop with origin $x_{0}$, hence an element of $\Omega_{x_{0}} X$. We may consider $P_{x_{0}} X$ as a principal bundle over $X$ with structure group $\Omega_{x_{0}} X$, and $\pi$ being the projection. Let us show that a closed 2-form $\omega$ on $X$, such that $\omega / 2 \pi$ represents an integer cohomology class, determines, up to an isomorphism, a principal $U(1)$ bundle $P$ over $X$.

Consider the action of the group $\Omega_{x_{0}} X$ on $U(1)$ defined by $\alpha^{\prime}=(\kappa) \alpha$, where $\kappa \in \Omega_{x_{0}} X$ and $\Delta(\kappa)=\exp \left(-i \int_{\sigma(\kappa)} \omega\right)$, with $\sigma(\kappa)$ being any 2-surface in $X$ such that $\partial \sigma=\kappa$ and $\alpha, \alpha^{\prime} \in U(1)$. Using this action, we build the associated bundle $P=$ $P_{x_{0}} X \times{ }_{\Delta} U(1)$ by identifying $(l, \alpha)$ with $\left(l \kappa, \Delta\left(\kappa^{-1}\right) \alpha\right) . P$ is a bundle with base $X$ and fibre $U(1)$. There is no longer any $\Omega_{x_{0}} X$ action on $P$ (since we have divided by this action!), but there is a free right $U(1)$ action defined by $[l, \alpha] \beta=[l, \alpha \beta]$. This proves that $P$ is a $U(1)$ principal bundle.

Acknowledgements. We would like to thank T. Januszkiewicz for discussions.

\section{References}

1. Killingback, T.: World-sheet anomalies and loop geometry. Nucl. Phys. B288, 578-588 (1987)

2. Witten, E.: Global anomalies in string theory. In: Anomalies, geometry and topology, ed. Bardeen, W., White, A.: Singapore pp. 61-99. World Scientific 1985

3. Alvarez-Gaumé, L.: Supersymmetry and the Atiyah-Singer index theorem. Commun. Math. Phys. 90, 161-173 (1983)

4. Pilch, K., Schellekenes, A. N., Warner, N. P.: Path integral calculation of string anomalies. Nucl. Phys. B287, 362-380 (1987)

5. Witten, E.: Elliptic genera and quantum field theory. Commun. Math. Phys. 109, 525-536 (1987)

6. Witten, E.: The index of the Dirac operator in loop space. Princeton preprint PUPT-1050 (1987)

7. Friedan, D., Windey, P.: Supersymmetric derivation of the Atiyah-Singer index theorem and chiral anomaly. Nucl. Phys. B235 (1984)

8. Pressley, A., Segal, G. B.: Loop groups. Oxford. Oxford University Press 1986

9. Moore, G., Nelson, P.: Anomalies in nonlinear sigma models. Phys. Rev. Lett. 53, 1519-1522 (1984)

10. Freed, D. S.: Determinants, torsion, and strings. Commun. Math. Phys. 107, 483-513 (1986)

11. Pilch, K., Warner, N. P.: String structures and the index of the Dirac-Ramond operator on orbifolds. Commun. Math. Phys. 115, 191-212 (1988)

12. Segal, G.: Elliptic cohomology. Sémin. Bourbaki, 40e année (1987-1988) \#495

13. Hamilton, R.: The inverse function theorem of Nash and Moser. Bull. Am. Math. Soc. 7, 65-222 (1982)

14. Milnor, J.: Remarks on infınıte-dimensıonal Lie groups. In: Relativity, groups and topology, II. (Les Houches) De Witt, B. S., Stora, R.: (eds.). Amsterdam: North Holland 1984

15. Switzer, R. M.: Algebraic topology-homotopy and homology. Berlin, Heidelberg, New York: Springer 1975

16. Freed, D. S.: The geometry of loop groups. Ph.D. thesis, Berkeley (1985)

17. Kac, V. G.: Infinite dimensional Lie algebras. Cambridge: Cambridge University Press 1985

18. Segal, G. B.: Unitary representations of some infinite dimensional groups. Commun. Math. Phys. 80, 301-342 (1981)

19. Kobayashi, S., Nomizu, K.: Foundations of differential geometry. vols. I and II, New York: WileyInterscience 1969

20. Chern, S. S., Simons, J.: Characteristic forms and geometric invariants. Ann. Math. 99, 48-69 (1974)

21. Bott, R., Tu, L. W.: Differential forms in algebraic topology. Berlin, Heidelberg, New York: Springer 1982 
22. Haefliger, A.: Sur l'extension du groupe structural d'un espace fibré. C. R. Acad. Sci. 243, 558-560 (1956)

23. Husemoller, D.: Fibre bundles. Berlin, Heidelberg, New York: Springer 1975

24. Steenrod, N. E.: The topology of fibre bundles. Princeton, NJ: Princeton University Press 1951; Witten, E.: Topological tools in ten dimensional physıcs. In: Unified strıng theories. Green, M., Gross, D.: (eds.) Singapore: World Scientific 1986

25. Grothendieck, A.: Sur quelques points d'algèbre homologique. Tohoku Math. J. 9, 119-221 (1957)

26. Borel, A., Hirzebruch, F.: Characteristic classes and homogeneous spaces, I. Am. J. Math. 80, 438 (1958)

Communicated by L. Alvarez-Gaumé

Received May 2, 1988; in revised form August 8, 1988 